\title{
Effect of photonic bandgap on luminescence from silicon nanocrystals
}

\author{
Ilya Sychugov, ${ }^{1}$ Niklas Elfström, ${ }^{1}$ Anders Hallén, ${ }^{1}$ Jan Linnros, ${ }^{1, *}$ and Min Qiu $^{2}$ \\ ${ }^{1}$ Laboratory of Materials and Semiconductor Physics, Royal Institute of Technology, SE-16440, Electrum 229, \\ Kista-Stockholm, Sweden \\ ${ }^{2}$ Laboratory of Optics, Photonics and Quantum Electronics, Royal Institute of Technology, SE-16440, Electrum 229, \\ Kista-Stockholm, Sweden \\ *Corresponding author: linnros@imit.kth.se
}

Received February 8, 2007; revised April 24, 2007; accepted May 9, 2007; posted May 10, 2007 (Doc. ID 79914); published June 21, 2007

\begin{abstract}
The modification of the luminescence of silicon nanocrystals experiencing the effect of a photonic bandgap in a $2 \mathrm{D}$ photonic crystal was investigated. The time-integrated photoluminescence spectra detected in the plane of the photonic crystal revealed a dip in the light emission corresponding to the wavelength of the bandgap, whose position changes according to the geometry of the prepatterned pillar array. The calculated emission pattern for a pointlike dipole placed in such a structure suggests an inhibition of the spontaneous emission rate at certain directions as a physical reason for the observed modification of luminescence. (C) 2007 Optical Society of America

OCIS code: 260.3800.
\end{abstract}

The properties of a light emitter can be modified by the properties of the radiation field around it. As a result, it was shown that a photonic crystal could provide ways to manipulate the spontaneous emission rate for an emitter placed into such a structure [1]. In general, the presence of the bandgap in a photonic crystal affects the spontaneous emission rate of an emitter by changing the density of optical modes, which in turn governs the rate of radiative transitions following Fermi's golden rule. Such an inhibition of spontaneous emission and corresponding lifetime increase can be of interest for certain applications when realized in a controllable way. The unwanted radiative recombination channels can be suppressed, thus leading to a redistribution of the light emission into more useful collectable radiative modes [2]. Another example is a photosensitization effect, where the energy is transferred by a near-field mechanism from an entity with a high optical absorption cross section to a less susceptible one, for instance from a quantum dot to a desired atomic species [3]. An increased lifetime of the excitation in a quantum dot can, therefore, enhance the probability of such an energy transfer process.

Introducing a defect into a photonic crystal bandgap structure, on the other hand, makes possible the formation of localized standing waves within the photonic lattice. If a pointlike emitter is placed into such a cavity an emitter-cavity interaction can be expected, leading to a shortening of the excitation lifetime [4]. In general, the degree of coupling depends on the proximity of emitter and cavity properties in wavelength and space domains: $Q / V$, where $Q$ is a quality factor and $V$ is the cavity volume. It was recently shown for direct bandgap nanocrystals that the highest cavity factor of any cavity type could be achieved using such photonic crystal defect structures. In the weak coupling regime, also referred to as the Purcell effect, a nearly 3 orders of magnitude increase in peak maximum intensity was reported
[5]. Even a strong coupling regime was demonstrated for such systems, where the energy is continually shifted back and forth between the electromagnetic field in the cavity and the exciton in the nanocrystal, the so-called Rabi oscillations [6].

Silicon nanocrystals are particularly interesting for optical applications because of their very-largescale integration (VLSI) compatibility. However, this type of quantum dot still possesses an indirect electronic bandgap structure similar to bulk $\mathrm{Si}$, which manifests in the long excitation lifetime of the order of microseconds [7]. Hence, there is an interest in finding ways to modify the excitation lifetime in such nanostructures to increase the light emission yield. A spatial redistribution of the light emission from $\mathrm{Si}$ nanocrystals placed in a photonic crystal was reported in [8]. In that case the collection efficiency of the light from light emitting diodes (LEDs) based on Si nanocrystals was enhanced in certain directions. The photonic lattice thus operated in a passive mode, similar to a diffraction grating. A direct modification of the light emission, on the other hand, for such nanocrystals was demonstrated when they were embedded into artificial opals, which represent a 3D photonic crystal structure with a pseudogap [9]. The photonic bandgap effect was, however, smeared in such a photonic lattice owing to size nonuniformity of the fused silica spheres and the lack of control in their positioning.

In this work we utilized electron-beam (e-beam) lithography to form well-defined $2 \mathrm{D}$ photonic crystal structures in fused silica doped with luminescent silicon nanocrystals with the aim of investigating the effect of a photonic bandgap on the light emission properties in detail. The goals were to show the dependence of the effect on photonic crystal bandgap parameters and to obtain the actual values of the emission inhibition that can be realized in a system with $\mathrm{Si}$ nanocrystals in a $\mathrm{SiO}_{2}$ matrix. We used a fused silica wafer instead of a thermally grown $\mathrm{SiO}_{2}$ 
layer on top of a silicon wafer in order to avoid possible interference of the emitted light with the reflected modes from such an interface.

Silicon nanocrystals were created by implantation of $\mathrm{Si}^{+}$ions with energy $270 \mathrm{keV}$ and fluence 2 $\times 10^{17} \mathrm{~cm}^{-2}$ into a fused silica wafer with subsequent annealing $\left(1100^{\circ} \mathrm{C}, 1 \mathrm{~h}\right)$ [7]. To form a photonic crystal lattice made of silica pillars, doped with luminescent silicon nanocrystals, a double-masking layer approach was used. First, an array of resist dots was defined by using e-beam lithography on top of a predeposited polycrystalline silicon (poly-Si) layer of $\sim 500 \mathrm{~nm}$ thickness. The resist dots served as a mask for reactive ion etching of the poly-Si layer, which, in turn, acted as a mask for the subsequent silicon dioxide etching. The arrays were designed such that the middle of the photonic bandgap falls within the broad peak of the luminescence spectrum of silicon nanocrystals (around $800 \mathrm{~nm}$ ). Time-integrated photoluminenscence (PL) measurements were carried out using a microphotoluminescence setup with the $325 \mathrm{~nm}$ line of a cw $\mathrm{He}-\mathrm{Cd}$ laser as the excitation source in geometry where the emitted light can be collected from the edge of the structures. A small numerical aperture objective lens was used $(\times 10$, NA $=0.3$ ) to selectively collect the in-plane emission

In Figs. 1(a) and 1(b) scanning electron microscopy (SEM) images of the manufactured photonic crystal of $\mathrm{SiO}_{2}$ pillars doped with $\mathrm{Si}$ nanocrystals are shown. The density of nanocrystals along the pillar height follows the implantation profile of $\mathrm{Si}$ atoms with a
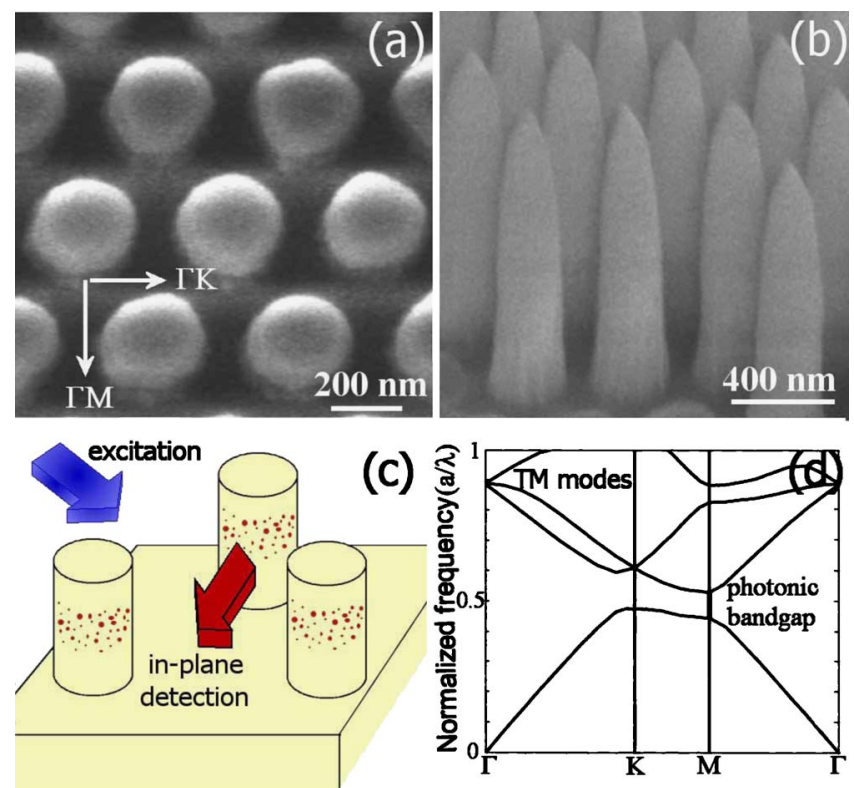

Fig. 1. (Color online) (a), (b) SEM images of the manufactured photonic crystal lattice. Fused silica pillars doped with luminescent Si nanocrystals were defined with e-beam lithography and etched by using reactive ion etching to form a triangular lattice. (c) Schematic representation of the geometry used in the PL experiment. Nanocrystals are excited by the $325 \mathrm{~nm}$ line of a He-Cd laser, and luminescence is detected in the plane of the photonic crystal, in the $\Gamma \mathrm{M}$ direction of the lattice. (d) Photonic bandgap calculations show the existence of a photonic bandgap in the $\Gamma \mathrm{M}$ direction for TM modes in such a structure. peak position $\sim 400 \mathrm{~nm}$ down from the top [Fig. 1(c)]. The vertical confinement of the modes is provided by the contrast in refractive index for the part of pillars oversaturated with $\mathrm{Si}$ (forming $\mathrm{Si}$ nanocrystals) ( $n$ $=1.65$ ) compared with the other parts with the refractive index of fused silica: $n_{\mathrm{SiO}_{2}}=1.45$ [10]. Such a structure possesses a photonic bandgap feature for TM modes, as seen from the bandgap calculations presented in Fig. 1(d). The 2D band structures were calculated by using the plane wave expansion method [11], where an effective refractive index 1.55 of the doped silica is used to account for the vertical confinement [12]. Here, as an example, the diameter of the pillars is chosen as $232 \mathrm{~nm}$, and the lattice constant $a=420 \mathrm{~nm}$.

The PL spectra detected from these structures are shown in Figs. 2(a) and 2(b). It is seen that there is a dip in light emission for in-plane radiation, whose position changes depending on the parameters of the lattice. While the lattice constant $a=420 \mathrm{~nm}$ was the same for all manufactured photonic crystals, the value of the pillar diameter was altered from $D$ $=200 \mathrm{~nm}$ to $D=240 \mathrm{~nm}$ with an $8 \mathrm{~nm}$ step. In Fig. 2(c) we plot positions of the middle of the observed dip in normalized wavelengths versus the normalized lattice constant. These experimental points fall in line with the prediction of the bandgap middle position as followed from the bandgap calculations [dotted line
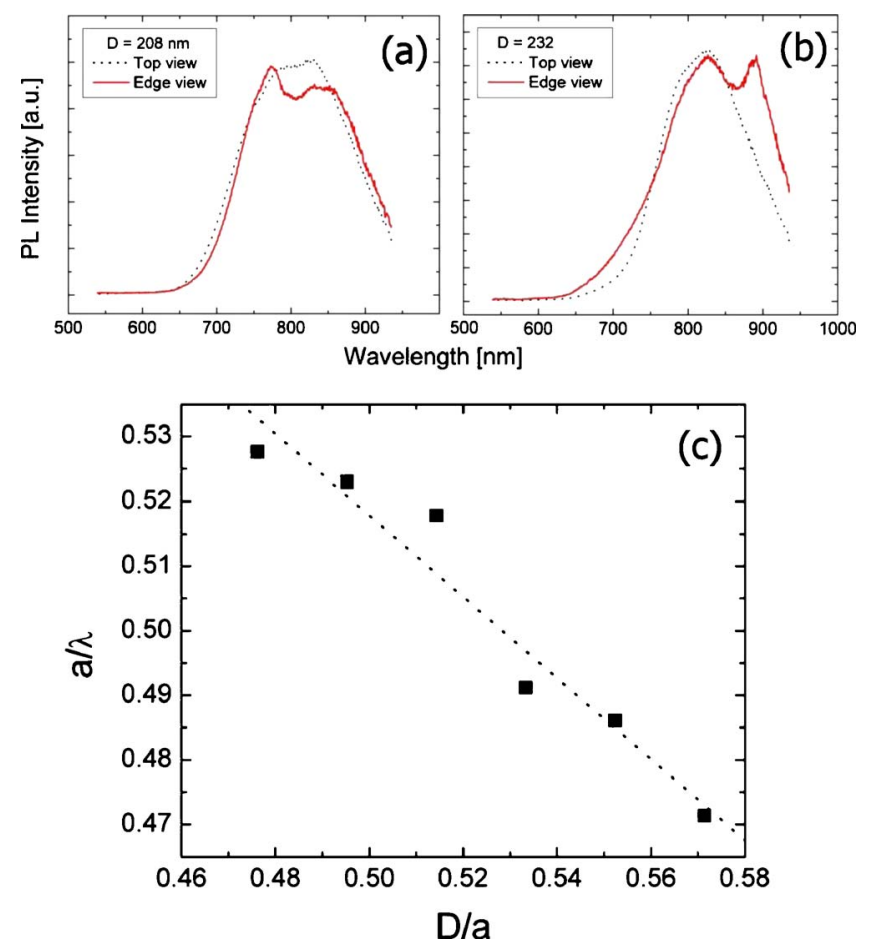

Fig. 2. (Color online) (a), (b) Measured PL spectra for Si nanocrystals in photonic lattices with different pillar diameters $D=208 \mathrm{~nm}$ and $D=232 \mathrm{~nm}$ and the same lattice constant $a=420 \mathrm{~nm}$. A dip in emission is seen for the case of in-plane detection of the emitted luminescence. (c) Squares are experimental values of the wavelength, corresponding to the middle of the dip in the detected in-plane PL spectra for various lattices. The dotted line is the result of bandgap calculations for the position of the middle of the bandgap [cf. Fig 1(d)]. 
in Fig. 2(c)]. The deviation of the experimental points from the calculated line can originate from the imperfections of manufactured pillar shape along with ambiguity of the central wavelength extraction from the broad dips on experimental curves. The approximative nature of the plane wave expansion method used here can also contribute to such a discrepancy.

For comparison, we have also performed full 3D simulations, using the finite-difference time-domain method [13] together with the perfectly matched layers [14] as the absorbing boundaries. The top view and the side view of the simulated structure are shown in Fig. 3(a). The continuous gradient of the refractive index of the doped silicon in the vertical direction is approximated by a step function with $50 \mathrm{~nm}$ intervals. A point dipole source emits a light pulse in the center of the photonic crystal. The pulse has a Gaussian-type spectrum, centered at the wavelength of $800 \mathrm{~nm}$. The calculated spectra for the emission of the dipole are shown in Figs. 3(b) and 3(c). It is seen that the value of the inhibition factor for TM modes is not high [Fig. 3(b)] and that there is no bandgap effect for the TE modes at all [Fig. 3(c)].

The observed difference in luminescence yield for emission at wavelengths falling inside and outside the bandgap is rather small ( $\sim 20 \%$, Fig. 2$)$. There are
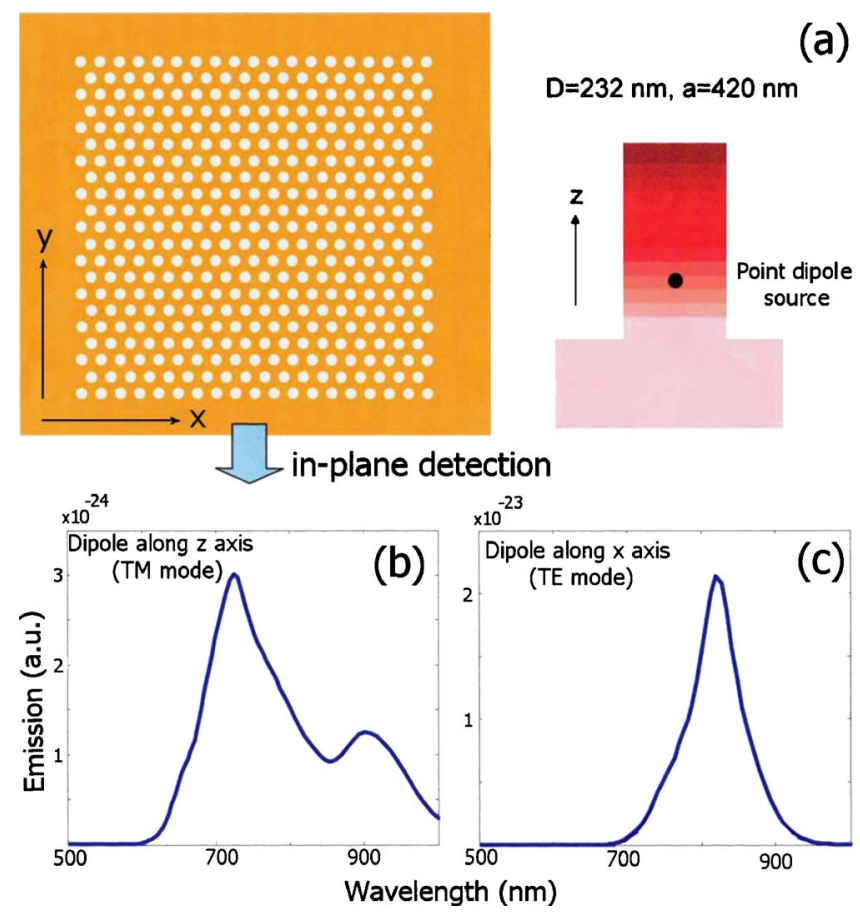

Fig. 3. (Color online) 3D calculations of the emission pattern of a point dipole placed into such a photonic crystal. (a) The refractive index profile along $z$ axis follows the implantation profile of $\mathrm{Si}$ atoms with a peak position of $n=1.65$ at $\sim 400 \mathrm{~nm}$ from the top [10]. Implanted $\mathrm{Si}$ atoms increase the refractive index of the fused silica $n_{\mathrm{SiO}_{2}}=1.45$ depending on the implantation dose. (b), (c) Emission spectra of the dipole for TM and TE modes for the in-plane detection in the $\Gamma \mathrm{M}$ direction. two main reasons for such an observation. First, the refractive index contrast between the structures $\left(n_{1}\right.$ $\approx 1.55)$ and the void $\left(n_{2}=1\right)$ in the present photonic crystal is not as high as, for example, in the case reported in [2], where air holes were made in a semiconductor slab with $n=3.5$. This fact is illustrated in Fig. 3(b), where only a small modification of the emission is predicted for the bandgap wavelengths. Second, the bandgap effect exists only for one type of polarization (cf. Fig. 3), when the dipole is oriented along the $z$ direction, while the measured signal in this experimental configuration is a combination of all possible polarizations of the emitted light.

In brief, we manufactured a photonic crystal bandgap structure with pillars of fused silica and embedded luminescent Si nanocrystals. The effect of the photonic bandgap on nanocrystal luminescence was observed. The measured parameters are in accordance with the calculated radiative pattern of a pointlike dipole placed into such a photonic crystal. The relatively small value of the effect is ascribed to the inherently small refractive index contrast between $\mathrm{SiO}_{2}$ and air in the given photonic lattice.

Support for this work was provided by the Swedish Research Council (VR). M. Qiu also acknowledges the support from the Swedish Foundation for Strategic Research (SSF) through the INGVAR program and the SSF Strategic Research Center in Photonics.

\section{References}

1. E. Yablonovitch, Phys. Rev. Lett. 58, 2059 (1987).

2. M. Fujita, S. Takahashi, Y. Tanaka, T. Asano, and S. Noda, Science 308, 1296 (2005).

3. D. Kovalev and M. Fujii, Adv. Mater. (Weinheim, Germany) 17, 2531 (2005).

4. P. Bhattacharya, J Sabarinathan, J Topolancik, S Chakravarty, P C. Yu, and W. Zhou Proc. IEEE 93, 1825 (2005).

5. A. Badolato, K. Hennesy, M. Atature, J. Dreiser, E. Hu, P. M. Petroff, and A. Imamoglu, Science 308, 1158 (2005).

6. T. Yoshie, A. Scherer, J. Hendrickson, G. Khitrova, H. M. Gibbs, G. Rupper, C. Eli, O. B. Schekin, and D. G. Deppe, Nature 432, 200 (2004).

7. J. Linnros, N. Lalic, A. Galeckas, and V. Grivickas, J. Appl. Phys. 86, 6128 (1999).

8. C. D. Presti, A. Irrera, G. Franzo, I. Crupi, F. Priolo, F. Iacona, G. Di Stefano, A. Piana, D. Sanfilippo, and P. G. Fallica, Appl. Phys. Lett. 88, 033501 (2006).

9. J. Valenta, J. Linnros, R. Juhasz, J.-L. Rehspringer, F. Huber, C. Hirlimann, S. Cheylan, and R. G. Elliman, J. Appl. Phys. 93, 4471 (2003).

10. J. Valenta, T. Ostatnicky, I. Pelant, R. G. Elliman, J. Linnros, and B. Honerlage, J. Appl. Phys. 96, 5222 (2004).

11. K. M. Ho, C. T. Chan, and C. M. Soukoulis, Phys. Rev. Lett. 65, 3152 (1990).

12. M. Qiu, Appl. Phys. Lett. 81, 1163 (2002).

13. K. S. Yee, IEEE Trans. Antennas Propag. 14, 302 (1966).

14. J. P. Berenger, J. Comput. Phys. 114, 185 (1994). 